Discussion Papers in Economics

\title{
Affine Maximizers in Domains with Selfish Valuations.
}

Swaprava Nath and Arunava Sen

November 2014

Discussion Paper 14-12

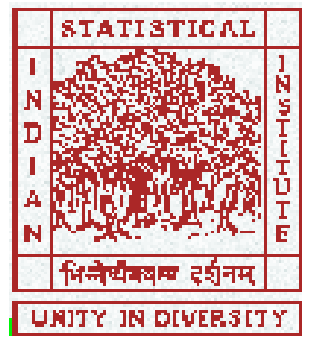

Indian Statistical Institute, Delhi

Economics and Planning Unit

7, S. J. S. Sansanwal Marg, New Delhi 110016, India 


\title{
Affine Maximizers in Domains With Selfish Valuations*
}

\author{
Swaprava Nath ${ }^{1}$ and Arunava Sen ${ }^{1}$ \\ ${ }^{1}$ Indian Statistical Institute, New Delhi
}

November 27, 2014

\begin{abstract}
We consider the domain of selfish and Lipschitz continuous preferences over a "rich" allocation space and show that onto, strategyproof and allocation non-bossy social choice functions are affine maximizers. Roberts (1979) proves this result for a finite set of alternatives and an unrestricted valuation space. In this paper, we show that in a sub-domain of the unrestricted valuations with the additional assumption of allocation non-bossiness, using the richness of the allocations, the strategyproof social choice functions can be shown to be affine maximizers. We provide an example to show that allocation non-bossiness is indeed critical for this result. This work shows that an affine maximizer result needs certain amount of richness split across valuations and allocations.
\end{abstract}

\section{INTRODUCTION}

Allocating divisible resources or tasks among competing agents is a classical economic problem. The proliferation of the Internet and the rapid development of computing power have created a marketplace for electronic resources. The consumers in these markets are typically individuals, small or medium businesses who pay the service providers for Internet connectivity. The frequencies of mobile telephony or $2 \mathrm{G} / 3 \mathrm{G}$ bandwidth are scarce resources and the businesses and service providers often have different valuations for the resources which are private information. Designing mechanisms that reveal the agents' demands truthfully is therefore an important problem. There is an extensive literature that deals with such applications (Yaïche et al., 2000; Sahasrabudhe and Kar, 2008; Wang et al., 2012). A similar resource allocation problem arises in the context of cloud computing and cloud storage.

All these markets use money extensively for their services, and agents privately observe their valuations or the costs of their tasks. Following the vast literature in this area, we use quasilinear utilities to model the payoffs. In addition, the valuations are private and selfish (an agent's

\footnotetext{
${ }^{*}$ We are grateful to Siddharth Chatterjee, Debasis Mishra and Shigehiro Serizawa for helpful discussions. We also thank two anonymous reviewers and the Associate Editor for valuable comments.
} 
valuation is a function of her own resource share). However, the valuations are not assumed to be increasing in the amount of the resources consumed. The following example shows that this situation occurs often in practice. Consider an agent who requires a certain amount of resource (to run her business optimally) say $\lambda \in[0,1]$ fraction of the divisible resource. However, there is a resource maintenance cost that increases proportionally with the allocated resource. As long as her allocation is $\delta(<\lambda)$, her returns are more than the cost and the valuation increases. But if she is allocated more than a share of $\lambda$, the cost of maintaining the resource is more than the returns and her valuation decreases. So the valuation has a peak in the amount of the resource share. In a similar way we can construct examples where multiples of a particular amount of resource share is desired by an agent and therefore the valuation can have multiple peaks.

The main result of this paper shows that the strategyproof and allocation non-bossy mechanisms in this model must belong to the well-known affine maximizer class. An immediate consequence of this result is that the payments are of a specific functional form.

In the following two subsections, we present the related literature and a panoramic sketch of the proof respectively.

\subsection{Relationship to THE Literature}

Our paper is related to two different strands in the literature. The first deals with results that characterize social choice functions (SCFs) that are affine maximizers. ${ }^{1}$ The original result appears in Roberts (1979). It considers a finite set of allocations $A$ (with $|A| \geq 3$ ) and an unrestricted valuation domain. According to the result, an onto and strategyproof SCF must be an affine maximizer. The case with $|A|=2$ differs significantly from the $|A| \geq 3$ case and is addressed in Marchant and Mishra (2014). They show that the class of onto, strategyproof SCFs expands to the class of generalized utility function maximizers. If the SCFs are required to satisfy an additional independence condition, this class reduces to the class of affine maximizers. Carbajal et al. (2013) extend this result to an infinite allocation space. They consider a compact set of allocations and unrestricted continuous valuations and show that the affine maximizer result continues to hold. All the results mentioned above require "sufficient" richness in the valuation space. In particular, the valuation of an agent is required to depend on the allocations of all agents and therefore not compatible with selfish valuations.

The assumption of selfish valuations constitutes a domain restriction; consequently the class of strategyproof SCFs expands beyond that of the class of affine maximizers. This has been shown in several papers. Lavi et al. (2003) considers combinatorial auction domains. They show that strategyproof SCFs are 'almost affine maximizers' provided they satisfy an independence of irrelevant alternatives assumption. Mishra and Quadir (2014) characterizes strategyproof SCFs for single object auctions. They show that this is a larger class than that of affine maximizers even with the non-bossiness assumption. There are other characterization results for restricted domains, e.g., Maya and Nisan (2012) for the two player cake-cutting problem, that show that the class of strategyproof SCFs is larger than that of the affine maximizer class.

\footnotetext{
${ }^{1}$ The term 'affine maximizer' was coined in Meyer-ter Vehn and Moldovanu (2002).
} 
Our paper is also related to the literature on the division model introduced in Sprumont (1991). In the single dimensional version of this model, a task has to be allocated amongst several agents. Monetary compensation is not allowed and valuations are assumed to be single-peaked. A salient allocation rule in this setting is uniform rule characterized in Sprumont (1991) and in several other papers using a variety of axioms (see, e.g., Ching (1994)). Recently, this model has been extended to multiple dimensions (Cho and Thomson, 2013; Morimoto et al., 2013).

Our paper differs from both strands of the literature with respect to the model and the results. We consider a task / object allocation space that is separable into allocations for each agent. On this domain, we consider arbitrary selfish valuations. The valuation space is a strict subclass of the unrestricted valuations, but is different from the sub-domains considered in the literature above. Furthermore, in contrast to the affine maximizer literature, we consider a 'rich' allocation space the set of allocations is a compact subset of $[0,1]^{n \times m}$. In contrast to the division model, singlepeakedness is replaced by monetary transfers in quasi-linear form.

In this model, we show that an onto, strategyproof SCF belongs to the class of affine maximizers if they satisfy the additional assumption of allocation non-bossiness. We observe that if 'richness' of the valuation domain is complemented with the 'richness' in the allocation space, then the affine maximizer result is restored. We believe that this observation is important and novel.

\subsection{BRIEF OVERVIEW OF THE PROOF}

The affine maximizer theorem in Roberts (1979) has been revisited several times with different proofs (Dobzinski and Nisan, 2009; Lavi et al., 2009; Mishra and Sen, 2012; Carbajal et al., 2013). Our proof builds on the arguments in Roberts (1979), Lavi et al. (2009) and Carbajal et al. (2013). The first two papers consider finite alternative space, while the last considers an uncountable compact alternative space with continuous valuations. As we have remarked earlier, all these papers require richness in the valuation space that is ruled out by the selfish valuations assumption. A critical element of these proofs is the construction of a value difference set for any two distinct allocations which is then shown to be a half-space. In the selfish valuation domain, this idea cannot be applied directly since there can exist two distinct allocations $x$ and $y$ whose $i$-th components $x_{i}$ and $y_{i}$ are identical. The value difference set for these two allocations will have zero at the $i$-th component for all vectors and will cease to have the half-space property.

In order to overcome this difficulty, we construct a subset of the allocation space called the distinct component set (DCS) that has distinct elements in each of the components. We show the existence of a maximal DCS and show that it is dense ${ }^{2}$ in the allocation space. Our proof proceeds by establishing the affine maximizer result on this maximal and dense DCS and then extending it to the full allocation space. The allocation non-bossiness assumption is used critically to establish the appropriate monotonicity condition required for the result.

The rest of the paper is organized as follows. We present the formal model in Section 2, state the main result in Section 3 and provide its proof in Section 4. We make a remark about the revenue equivalence result in Section 5. In Section 6, we discuss a more general result that can be

\footnotetext{
${ }^{2} \mathrm{~A}$ set $D$ is dense in a metric space $X$, if every $x \in X$ either belongs to $D$ or is a limit point of $D$.
} 
proved using the same proof. We conclude the paper in Section 7.

\section{The Model And Definitions}

Let $N=\{1, \ldots, n\}, n \geq 2$ be the set of agents. There is a set $J=\{1, \ldots, m\}$ of perfectly divisible tasks (or objects) to be allocated among the agents. Let $x_{i j} \in[0,1], i \in N, j \in J$ be the fraction of task $j$ assigned to agent $i$. We assume that each task is fully allocated, i.e., $\sum_{i \in N} x_{i j}=1, \forall j \in J$. Let $x_{i} \equiv\left(x_{i 1}, \ldots, x_{i m}\right) \in[0,1]^{m}$ denote the fractions of the tasks allocated to agent $i$. An allocation $x$ is therefore a matrix where the rows and columns correspond to the agents and tasks respectively. The $(i, j)$-th element of this matrix is $x_{i j}$, as explained above. Formally, we define the set of allocations to be:

$$
A=\left\{z \in[0,1]^{n \times m}: \sum_{i \in N} z_{i j}=1, \forall j \in J\right\}
$$

Observe that the allocations in $A$ are separable, i.e., it can be split into allocations for each agent, and is compact.

A valuation function for agent $i$ is a Lipschitz continuous ${ }^{3}$ map $u_{i}: A \rightarrow \mathbb{R}$. It is selfish if, $u_{i}\left(x_{i}, x_{-i}\right)=u_{i}\left(x_{i}, x_{-i}^{\prime}\right)$, for all $\left(x_{i}, x_{-i}\right),\left(x_{i}, x_{-i}^{\prime}\right) \in A$. Let $x \in A$. If $u_{i}$ is selfish, we can write $u_{i}(x)=u_{i}\left(x_{i}\right)$ without loss of generality. Selfishness implies that the valuation of an agent depends only on the task portions assigned to her, and does not depend on the task portions assigned to other agents.

Definition 1 (Arbitrary Selfish Valuations) The set of all selfish valuations is the domain of arbitrary selfish valuations $(A S V)$. It is denoted by $U$.

We do not put any further restrictions on valuation functions. In particular, we do not assume that the valuation function is increasing (i.e., $u_{i}\left(x_{i}\right) \geq u_{i}\left(x_{i}^{\prime}\right)$ whenever $x_{i}>x_{i}^{\prime}{ }^{4}$ ) or additively separable across tasks (i.e., for every agent $i$ there exists $u_{i j}:[0,1] \rightarrow \mathbb{R}$ for every task $j$ such that $\left.u_{i}\left(x_{i}\right)=\sum_{j \in J} u_{i j}\left(x_{i j}\right)\right)$. Note that imposing these conditions would restrict the domain beyond ASV. We are not sure whether the affine maximizer result continues to hold in these further restricted domains.

In addition to the utility that an agent derives from the tasks assigned to her, she can also be compensated with (or charged) money. Moreover, the overall utility function is quasi-linear in money, i.e., given by $u_{i}\left(x_{i}\right)+p_{i}$, where $p_{i}$ is agent $i$ 's monetary compensation.

We assume that the agent valuations are private information and must be elicited. The goal of the paper is to identify collective outcomes that induce agents to reveal their private information truthfully. The following definitions are standard in mechanism design literature.

Definition 2 (Social Choice Function) $A$ social choice function ( $S C F$ ) $F$ is a mapping from the set of valuation profiles to the set of allocations, i.e., $F: U^{n} \rightarrow A$.

\footnotetext{
${ }^{3}$ A function $f: A \subseteq \mathbb{R}^{m} \rightarrow \mathbb{R}$ is Lipschitz continuous if $\exists K \in \mathbb{R}$ such that for all $x, y \in A,|f(x)-f(y)| \leq K\|x-y\|$.

${ }^{4}$ Here $x_{i}>x_{i}^{\prime} \Rightarrow x_{i j}>x_{i j}^{\prime}$, for all $j \in J$.
} 
Definition 3 (Strategyproofness) A SCF F is strategyproof if there exist transfers $p_{i}: U^{n} \rightarrow$ $\mathbb{R}, i \in N$, such that for all $u=\left(u_{i}, u_{-i}\right) \in U^{n}$,

$$
u_{i}\left(F\left(u_{i}, u_{-i}\right)\right)+p_{i}\left(u_{i}, u_{-i}\right) \geq u_{i}\left(F\left(u_{i}^{\prime}, u_{-i}\right)\right)+p_{i}\left(u_{i}^{\prime}, u_{-i}\right), \quad \forall u_{i}^{\prime} \in U, i \in N .
$$

A strategyproof SCF induces truth-telling in dominant strategies.

The problem of characterizing strategyproof SCFs in allocation problems with selfish preferences presents special difficulties because agents can affect the allocations of other agents without affecting their own. An axiom that addresses this issue in a "minimal" way is that of allocation non-bossiness. The non-bossiness axiom was introduced in Satterthwaite and Sonnenschein (1981), and has been used widely in the literature on incentive compatibility. ${ }^{5}$

Definition 4 (Allocation Non-Bossiness) An SCF F satisfies allocation non-bossiness (ANB) if $\forall i \in N, \forall u_{-i} \in U^{n-1}$ and $\forall u_{i}, u_{i}^{\prime} \in U$ with $F_{i}\left(u_{i}, u_{-i}\right)=F_{i}\left(u_{i}^{\prime}, u_{-i}\right)$ implies $F\left(u_{i}, u_{-i}\right)=$ $F\left(u_{i}^{\prime}, u_{-i}\right)$, where $F_{i}(\cdot)$ is the $i$-th component of $F(\cdot)$.

Suppose an agent's allocation is unaffected by a change in her valuation. According to ANB, this must not change the allocation of any other agent. A well-known fact about strategyproofness is that a change in an agent's valuation that does not affect her allocation, does not also affect her payment. Therefore, the "if" part of non-bossiness together with strategyproofness ensures that the change in the agent's valuation does not change her overall utility. In such a situation, the allocations of other agents is not allowed to change. We place no restrictions on the payments of the other agents, so that their utilities may change.

It is important to distinguish our version of non-bossiness from a related notion which we refer to as outcome non-bossiness. In a quasi-linear domain, an outcome comprises of the allocation and payments. An SCF is outcome non-bossy if the following holds: if an agent does not change her outcome by changing her own valuation, she does not change the outcomes of the other agents.

Outcome non-bossiness and strategyproofness imply allocation non-bossiness. Outcome nonbossiness is therefore a stronger requirement than allocation non-bossiness. In fact, affine maximizers (defined below), in general, do not satisfy outcome non-bossiness.

A salient class of SCFs introduced in Roberts (1979) is that of affine maximizers, defined as follows.

Definition 5 (Affine Maximizers) An SCF $F: U^{n} \rightarrow A$ belongs to the class of affine maximizers if there exists $w_{i} \geq 0, i \in N$, not all zero, and a continuous function $\kappa: A \rightarrow \mathbb{R}$ such that,

$$
F(u) \in \underset{x \in A}{\operatorname{argmax}}\left(\sum_{i \in N} w_{i} u_{i}(x)+\kappa(x)\right) .
$$

The continuity of the valuations and the compactness of the set of allocations ensures that the class of affine maximizers is non-empty.

\footnotetext{
${ }^{5}$ For matching, see e.g., (Svensson, 1999; Pápai, 2000), and for exchange economies, see e.g., (Goswami et al., 2013; Momi, 2013). For a comprehensive discussion of the non-bossiness axiom, see Thomson (2014).
} 


\section{MAin Result}

In this section, we present the central result of the paper.

Theorem 1 (Affine Maximizers for Selfish Valuations) If $n \geq 3$, every onto ${ }^{6}$, ANB and strategyproof $S C F F: U^{n} \rightarrow A$ is an affine maximizer. If $n=2$, the result holds without the ANB assumption.

The ASV assumption ensures that onto affine maximizers exist. Our next claim shows the existence of affine maximizers with the properties mentioned in Theorem 1 . The proof is deferred to the Appendix.

Claim 1 There exist affine maximizers that are onto, strategyproof and ANB.

The ANB assumption is necessary for our result. We provide an example to show the existence of non-affine maximizer SCFs that are strategyproof and bossy.

EXAmple 1 (Serial Dictator with a Threshold) For simplicity, we illustrate a SCF F with three agents, $N=\{1,2,3\}$ and one task, i.e., $m=1$ (see Figure 1). The arguments can clearly be extended to a setting with arbitrary $n$ and $m$. Let $t$ be an arbitrary real number referred to as the threshold. The SCF F is defined below.

For any valuations profile $\left(u_{1}, u_{2}, u_{3}\right)$, the $S C F$ picks $x_{1}^{*} \in \operatorname{argmax}_{x_{1} \in[0,1]} u_{1}\left(x_{1}\right)$. Ties are broken in favor of the largest value of $x_{1}^{*}$. In order to determine the allocations of agents 2 and 3 , we need to consider the two following cases.

Case 1: $u_{1}\left(x_{1}^{*}\right) \leq t$ : Then $x_{2}^{*} \in \operatorname{argmax}_{x_{2} \in\left[0,1-x_{1}^{*}\right]} u_{2}\left(x_{2}\right)$ with ties broken as before, and $x_{3}^{*}=1-x_{1}^{*}-x_{2}^{*}$.

Case 2: $u_{1}\left(x_{1}^{*}\right)>t$ : In this case, the role of agents 2 and 3 is reversed, i.e., $x_{3}^{*} \in$ $\operatorname{argmax}_{x_{3} \in\left[0,1-x_{1}^{*}\right]} u_{3}\left(x_{3}\right)$ with ties broken as before, and $x_{2}^{*}=1-x_{1}^{*}-x_{3}^{*}$.

Clearly $F$ is onto. It is bossy because agent 1 can change the allocations of agents 2 and 3 without changing her own allocation. This is illustrated in Figure 1 - for the valuation profiles $\left(u_{1}, u_{2}, u_{3}\right)$ and $\left(u_{1}^{\prime}, u_{2}, u_{3}\right)$, the allocations are $\left[\begin{array}{lll}0.5 & 0.3 & 0.2\end{array}\right]^{\top}$ and $\left[\begin{array}{llll}0.5 & 0.2 & 0.3\end{array}\right]^{\top}$ respectively.

Strategyproofness of $F$ follows from standard arguments. We claim that $F$ is not an affine maximizer. To see this, we repeat the arguments from Equations 8 to 9 with $x=\left[\begin{array}{lll}0.5 & 0.3 & 0.2\end{array}\right]^{\top}$ and $y=\left[\begin{array}{lll}0.5 & 0.2 & 0.3\end{array}\right]^{\top}$ to get,

$$
\begin{aligned}
& w_{2} u_{2}(0.3)+w_{3} u_{3}(0.2)+\kappa\left(\left[\begin{array}{lll}
0.5 & 0.3 & 0.2
\end{array}\right]^{\top}\right)=w_{2} u_{2}(0.2)+w_{3} u_{3}(0.3)+\kappa\left(\left[\begin{array}{lll}
0.5 & 0.2 & 0.3
\end{array}\right]^{\top}\right) \\
\Rightarrow & \kappa\left(\left[\begin{array}{lll}
0.5 & 0.3 & 0.2
\end{array}\right]^{\top}\right)=\kappa\left(\left[\begin{array}{lll}
0.5 & 0.2 & 0.3
\end{array}\right]^{\top}\right) \quad \text { since } u_{2}(0.3)=u_{2}(0.2), u_{3}(0.2)=u_{3}(0.3) .
\end{aligned}
$$

Pick $u_{3}^{\prime}$ which agrees with $u_{3}$ everywhere except $[0.15,0.25]$ and is shown by the dashed line in the figure. By definition, $F\left(u_{1}, u_{2}, u_{3}^{\prime}\right)=\left[\begin{array}{lll}0.5 & 0.3 & 0.2\end{array}\right]^{\top}$. However,

\footnotetext{
${ }^{6}$ An SCF $F: U^{n} \rightarrow A$ is onto, if for all $x \in A$, there exists $u \in U^{n}$ such that $F(u)=x$.
} 


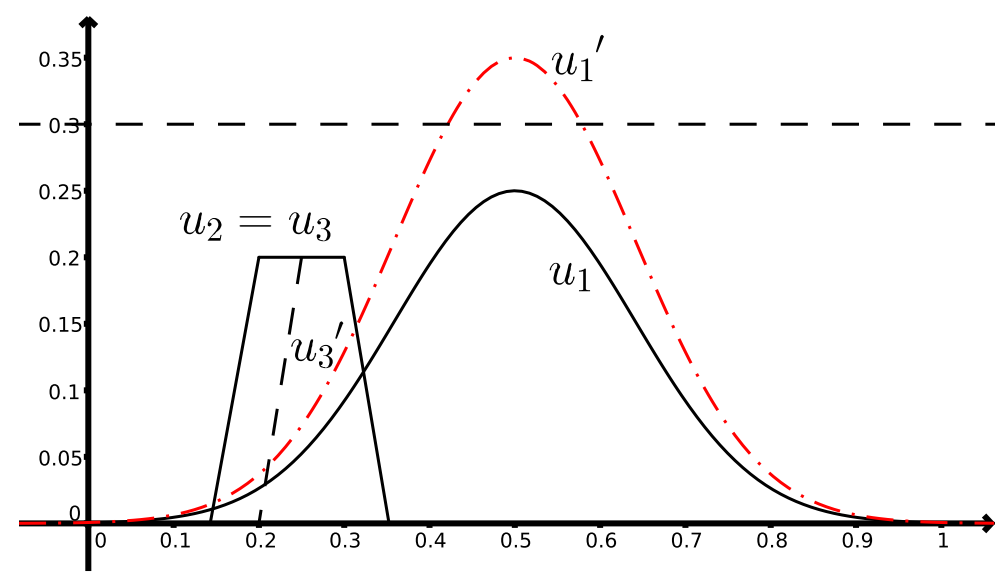

Figure 1: An example to illustrate the necessity of ANB.

$$
\begin{aligned}
& w_{1} u_{1}(0.5)+w_{2} u_{2}(0.3)+w_{3} u_{3}^{\prime}(0.2)+\kappa\left(\left[\begin{array}{lll}
0.5 & 0.3 & 0.2
\end{array}\right]^{\top}\right) \\
& <w_{1} u_{1}(0.5)+w_{2} u_{2}(0.2)+w_{3} u_{3}^{\prime}(0.3)+\kappa\left(\left[\begin{array}{lll}
0.5 & 0.2 & 0.3
\end{array}\right]^{\top}\right),
\end{aligned}
$$

since $u_{2}(0.2)=u_{2}(0.3)=0.2$ but $0.2=u_{3}^{\prime}(0.3)>u_{3}^{\prime}(0.2)=0$ and $\kappa$ is same at these two allocations. Hence, $F$ is not an affine maximizer.

\section{Proof of the TheOrem}

In order to present the proof, we need to define an important property. Since allocations are decomposable into components for every agent and valuations are selfish, it is possible for the component of agent $i$ to be identical in two different allocations. To account for this possibility we need the following modification of the notion of positive association of differences (PAD) which appears in Roberts (1979).

Definition 6 (PAD-DC) An SCF F satisfies positive association of differences for distinct components (PAD-DC) if $\forall u, u^{\prime} \in U^{n}$,

$$
\left[F(u)=x \text { and } u_{i}^{\prime}\left(x_{i}\right)-u_{i}\left(x_{i}\right)>u_{i}^{\prime}\left(y_{i}\right)-u_{i}\left(y_{i}\right), \forall y \in A \backslash\{x\} \text { and } y_{i} \neq x_{i}, \forall i \in N\right] \Rightarrow\left[F\left(u^{\prime}\right)=x\right] \text {. }
$$

Let $x$ be an allocation and $u$ be a valuation profile such that $F(u)=x$. Such a profile exists because of the onto-ness of $F$. If we consider all other allocations in $A$, there will be an allocation $z \in A$ such that $x_{j}=z_{j}$ for some $j \in N$. Since valuations are selfish, $u_{j}^{\prime}\left(x_{j}\right)-u_{j}\left(x_{j}\right)=u_{j}^{\prime}\left(z_{j}\right)-u_{j}\left(z_{j}\right)$ and the conclusion $F\left(u^{\prime}\right)=x$ cannot be inferred from PAD. However, if $u_{i}^{\prime}\left(x_{i}\right)-u_{i}\left(x_{i}\right)>u_{i}^{\prime}\left(y_{i}\right)-$ $u_{i}\left(y_{i}\right), \forall y_{i} \neq x_{i}$, we can conclude $F\left(u^{\prime}\right)=x$ from PAD-DC. Of course, for the ASV domain, PAD-DC implies PAD, i.e., PAD-DC is a stronger property than PAD.

The next result shows that PAD-DC is an implication of ANB and strategyproofness. 
Lemma 1 If $n \geq 3$, every ANB, strategyproof $S C F$ satisfies $P A D-D C$. For $n=2$, every strategyproof $S C F$ satisfies $P A D-D C$.

Proof: We prove the lemma in three steps. Let $F$ be strategyproof and ANB.

Step 1: Pick any $i \in N$ and let $\left(u_{i}, u_{-i}\right),\left(u_{i}^{\prime}, u_{-i}\right) \in U^{n}$ be such that $F(u)=x$, and suppose $\forall y \in A \backslash\{x\}, u_{i}^{\prime}\left(x_{i}\right)-u_{i}\left(x_{i}\right)>u_{i}^{\prime}\left(y_{i}\right)-u_{i}\left(y_{i}\right)$, for all $y_{i} \neq x_{i}$. We claim $F_{i}\left(u_{i}^{\prime}, u_{-i}\right)=x_{i}$.

Suppose not, i.e., $F_{i}\left(u_{i}^{\prime}, u_{-i}\right)=z_{i} \neq x_{i}$. By assumption, $u_{i}^{\prime}\left(x_{i}\right)-u_{i}\left(x_{i}\right)>u_{i}^{\prime}\left(z_{i}\right)-u_{i}\left(z_{i}\right)$. However, strategyproofness of $F$ implies $u_{i}^{\prime}\left(x_{i}\right)-u_{i}\left(x_{i}\right) \leq u_{i}^{\prime}\left(z_{i}\right)-u_{i}\left(z_{i}\right)^{7}$, which is a contradiction. Hence $F_{i}\left(u_{i}^{\prime}, u_{-i}\right)=x_{i}$.

Step 2: Consider the case $n \geq 3$. Since Step 1 is true for every $i \in N, u_{i}, u_{i}^{\prime} \in U, u_{-i} \in U^{n-1}$, ANB implies that $F\left(u_{i}^{\prime}, u_{-i}\right)=x$. If $n=2$, it is trivially true since there are only two components in each allocation $x_{1}$ and $x_{2}$, which are row vectors of length $m$ and their sums equal the 'all-one' vector of length $m$. Hence, if $x_{1}$ does not change, nor does $x_{2}$.

Step 3: We use these arguments for every player. Fix $u, u^{\prime} \in U^{n}$ as in the definition of PAD-DC, i.e., $F(u)=x$ and $u_{i}^{\prime}\left(x_{i}\right)-u_{i}\left(x_{i}\right)>u_{i}^{\prime}\left(y_{i}\right)-u_{i}\left(y_{i}\right), \forall y \in A \backslash\{x\}, y_{i} \neq x_{i}, \forall i \in N$. We have shown that $F\left(u_{i}, u_{-i}\right)=x$ implies $F\left(u_{i}^{\prime}, u_{-i}\right)=x$. We repeat Steps 1 and 2 above for the transition from the value profile $\left(u_{i}^{\prime}, u_{-i}\right)$ to $\left(u_{i}^{\prime}, u_{j}^{\prime}, u_{-i j}\right)$ and conclude that $F\left(u_{i}^{\prime}, u_{j}^{\prime}, u_{-i j}\right)=x$. Proceeding in this manner we conclude that $F\left(u^{\prime}\right)=x$, as needed.

The next claim will be used repeatedly in the proof. It is a counterpart of Claim 1 in Lavi et al. (2009). Their argument cannot be used directly because of the domain restriction used in this paper.

Claim 2 Let $F$ satisfy PAD-DC, and fix $u, u^{\prime} \in U^{n}$. Then,

$$
\left[F(u)=x \text { and } u_{i}^{\prime}\left(x_{i}\right)-u_{i}^{\prime}\left(y_{i}\right)>u_{i}\left(x_{i}\right)-u_{i}\left(y_{i}\right) \text { whenever } y_{i} \neq x_{i}, \forall i \in N\right] \Rightarrow\left[F\left(u^{\prime}\right) \neq y\right]
$$

Proof: Suppose not, i.e., $F\left(u^{\prime}\right)=y$. We will construct a valuation $u_{i}^{\prime \prime} \in U, i \in N$ such that an application of PAD-DC to the transition $u^{\prime} \rightarrow u^{\prime \prime}$ will yield $F\left(u^{\prime \prime}\right)=y$. On the other hand, its application to the transition $u \rightarrow u^{\prime \prime}$ would imply $F\left(u^{\prime \prime}\right)=x$, leading to a contradiction.

We need to construct $u^{\prime \prime}$ to satisfy:

$$
u_{i}^{\prime \prime}\left(y_{i}\right)-u_{i}^{\prime}\left(y_{i}\right)>u_{i}^{\prime \prime}\left(z_{i}\right)-u_{i}^{\prime}\left(z_{i}\right), \forall z_{i} \neq y_{i}, \forall i \in N
$$

and,

$$
u_{i}^{\prime \prime}\left(x_{i}\right)-u_{i}\left(x_{i}\right)>u_{i}^{\prime \prime}\left(z_{i}\right)-u_{i}\left(z_{i}\right), \forall z_{i} \neq x_{i}, \forall i \in N .
$$

\footnotetext{
${ }^{7}$ Let $p_{i}(\cdot), i \in N$ be the payment function that makes $F$ strategyproof. The required inequality is obtained by summing the following inequalities which are consequences of strategyproofness:

$$
\begin{aligned}
& u_{i}\left(x_{i}\right)+p_{i}\left(u_{i}, u_{-i}\right) \geq u_{i}\left(z_{i}\right)+p_{i}\left(u_{i}^{\prime}, u_{-i}\right), \\
& u_{i}^{\prime}\left(z_{i}\right)+p_{i}\left(u_{i}^{\prime}, u_{-i}\right) \geq u_{i}^{\prime}\left(x_{i}\right)+p_{i}\left(u_{i}, u_{-i}\right) .
\end{aligned}
$$
}


Pick a pair of allocations $x$ and $y$ such that the LHS of the implication of the claim occurs, i.e., $F(u)=x$ and $u_{i}^{\prime}\left(x_{i}\right)-u_{i}^{\prime}\left(y_{i}\right)>u_{i}\left(x_{i}\right)-u_{i}\left(y_{i}\right)$ whenever $y_{i} \neq x_{i}, \forall i \in N$. A simple rearrangement of the terms yields, $\beta=u_{i}\left(x_{i}\right)-u_{i}^{\prime}\left(x_{i}\right)<u_{i}\left(y_{i}\right)-u_{i}^{\prime}\left(y_{i}\right)=\alpha$. Let $\delta=\alpha-\beta$ which is positive by assumption. The difference of this $u_{i}^{\prime \prime}$ from that in Lavi et al. (2009) is that the construction here must also satisfy Lipschitz continuity of $u_{i}^{\prime \prime}$. We will choose $u_{i}^{\prime \prime}$ such that (a) $u_{i}^{\prime \prime}\left(x_{i}\right)-u_{i}^{\prime}\left(x_{i}\right)=\beta+\delta / 4$ and (b) $u_{i}^{\prime \prime}\left(y_{i}\right)-u_{i}^{\prime}\left(y_{i}\right)=\alpha-\delta / 4$.

We show that it is possible to construct such a $u_{i}^{\prime \prime}$ in the ASV domain using the fact that any arbitrary selfish valuation is admissible. There are only three possibilities: (1) $\alpha>\beta>0,(2)$ $\alpha>0, \beta<0$, and (3) $0>\alpha>\beta$. Since $u_{i}, u_{i}^{\prime}$ are continuous functions, they must have minima over $[0,1]^{m}$. Denote these by $m, m^{\prime}$ respectively, and let $\bar{m}=\min \left\{m, m^{\prime}\right\}$. Define:

$$
\begin{aligned}
& s=\sup _{x_{i} \in[0,1]^{m}} \sup _{\{\vec{e}:\|\vec{e}\|=1\}} \max \left\{\lim _{t \rightarrow 0-}\left|\frac{\left.u_{i}\left(x_{i}+t \vec{e}\right)-u_{i}\left(x_{i}\right)\right)}{t}\right|, \lim _{t \rightarrow 0+}\left|\frac{\left.u_{i}\left(x_{i}+t \vec{e}\right)-u_{i}\left(x_{i}\right)\right)}{t}\right|\right\} \\
& s^{\prime}=\sup _{x_{i} \in[0,1]^{m}} \sup _{\{\vec{e}:\|\vec{e}\|=1\}} \max \left\{\lim _{t \rightarrow 0-}\left|\frac{\left.u_{i}^{\prime}\left(x_{i}+t \vec{e}\right)-u_{i}^{\prime}\left(x_{i}\right)\right)}{t}\right|, \lim _{t \rightarrow 0+}\left|\frac{\left.u_{i}^{\prime}\left(x_{i}+t \vec{e}\right)-u_{i}^{\prime}\left(x_{i}\right)\right)}{t}\right|\right\},
\end{aligned}
$$

where $\vec{e}$ is a unit vector in $\mathbb{R}^{m}$. Therefore $s$ and $s^{\prime}$ are the maximum rates of change of the functions $u_{i}$ and $u_{i}^{\prime}$. Since $u_{i}, u_{i}^{\prime}$ are Lipschitz continuous, the directional derivatives of the functions are bounded at any point $x$. Hence the limits in the above equations will be finite, i.e., $s, s^{\prime}<\infty$. Let $s=\max \left\{s, s^{\prime}\right\}$. In order to construct $u_{i}^{\prime \prime}$, we proceed as follows. Let,

$$
u_{i}^{\prime \prime}\left(z_{i}\right)= \begin{cases}u_{i}^{\prime}\left(x_{i}\right)+\beta+\delta / 4 & \text { for } z_{i}=x_{i} \\ u_{i}^{\prime}\left(y_{i}\right)+\alpha-\delta / 4 & \text { for } z_{i}=y_{i}\end{cases}
$$

Define,

$$
\bar{s}\left(x_{i}, y_{i}, s\right)=\max \left\{\frac{\left|u_{i}^{\prime \prime}\left(x_{i}\right)-(\bar{m}-\delta)\right|}{|| x_{i}-y_{i}|| / 2}, \frac{\left|u_{i}^{\prime \prime}\left(y_{i}\right)-(\bar{m}-\delta)\right|}{|| x_{i}-y_{i}|| / 2}, s\right\} .
$$

With this definition, pick $\hat{s}>\bar{s}$. We now complete the specification of the function $u_{i}^{\prime \prime}$ :

$$
u_{i}^{\prime \prime}\left(z_{i}\right)= \begin{cases}u_{i}^{\prime}\left(x_{i}\right)+\beta+\delta / 4 & \text { for } z_{i}=x_{i} \\ u_{i}^{\prime}\left(y_{i}\right)+\alpha-\delta / 4 & \text { for } z_{i}=y_{i} \\ u_{i}^{\prime \prime}\left(x_{i}\right)-\hat{s}\left\|z_{i}-x_{i}\right\| & \text { for } z_{i}:\left\|z_{i}-x_{i}\right\| \leq\left(u_{i}^{\prime \prime}\left(x_{i}\right)-(\bar{m}-\delta)\right) / \hat{s} \\ u_{i}^{\prime \prime}\left(y_{i}\right)-\hat{s}\left\|z_{i}-y_{i}\right\| & \text { for } z_{i}:\left\|z_{i}-y_{i}\right\| \leq\left(u_{i}^{\prime \prime}\left(y_{i}\right)-(\bar{m}-\delta)\right) / \hat{s} \\ \bar{m}-\delta & \text { otherwise }\end{cases}
$$

The construction has the following features:

1. It has a 'conical' peak both at $x_{i}$ and $y_{i}$.

2. The slopes of $u_{i}^{\prime \prime}$ around $x_{i}$ and $y_{i}$ are chosen so that the difference function $u_{i}^{\prime \prime}-u_{i}^{\prime}$ is maximized at $y_{i}$ and $u_{i}^{\prime \prime}-u_{i}$ is maximized at $x_{i}$ within these conical regions.

3. It is flat outside these conical regions and is small enough so that $u_{i}^{\prime \prime}-u_{i}^{\prime}$ at these points are smaller than that at $y_{i}$ and $u_{i}^{\prime \prime}-u_{i}$ at these points are smaller than that at $x_{i}$. This ensures that $y_{i}$ and $x_{i}$ are the global maximizers of the difference functions $u_{i}^{\prime \prime}-u_{i}^{\prime}$ and $u_{i}^{\prime \prime}-u_{i}$ respectively. 
According to the construction, $u_{i}^{\prime \prime}$ satisfies Equations (1) and (2). Also, the supremum of all possible directional derivatives of $u_{i}^{\prime \prime}$ is $\hat{s}$. Hence $u_{i}^{\prime \prime}$ is Lipschitz continuous. This concludes the proof.

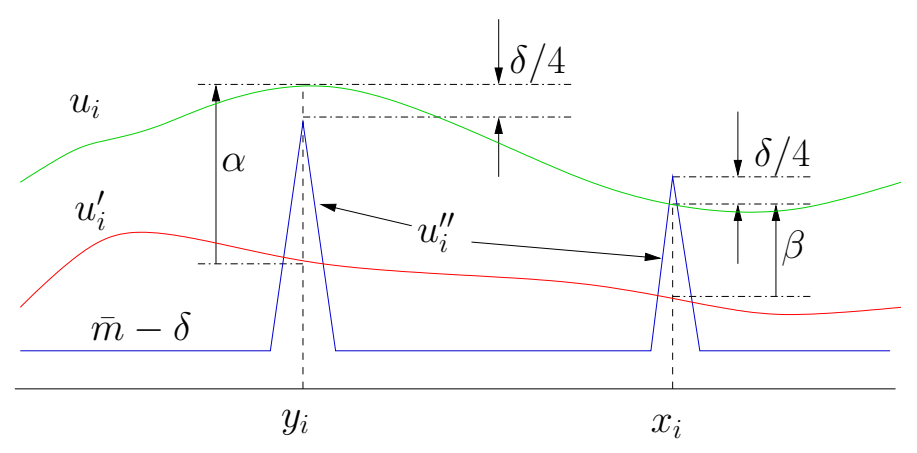

Figure 2: Illustration of an example construction (Claim 2).

Figure 2 illustrates an example construction of $u_{i}^{\prime \prime}$ for a specific $u_{i}$ and $u_{i}^{\prime}$ and $m=1$.

One of the central ideas of our proof is to show that $F$ is an affine maximizer on a suitably chosen dense subset of the allocation set $A$. The proof is completed by extending the result to the entire set of allocations using the continuity of the valuations. The following definition is critical for the choice of the dense subset.

Definition 7 (Distinct Component Set) $A$ set $S \subseteq A$ is called a distinct component set (DCS) if for every distinct $x, y \in S, x_{i} \neq y_{i}, \forall i \in N$.

We consider the DCSs that contain a specific allocation $x$. Denote the set of all DCSs containing $x$ by $\mathscr{D}_{x}$. Our next result shows that a Maximal and Dense DCS (MD-DCS) exists in $\mathscr{D}_{x}$.

Proposition 1 For every $x \in A$, there exists a maximal element in $\mathscr{D}_{x}$ which is dense in $A$.

The proof of this proposition is deferred to the Appendix.

Pick an arbitrary $x \in A$ and an MD-DCS $D_{x}$. All the following results until Claim 10 refer to $x$ and $D_{x}$. Claims 3 to 11 have counterparts in Lavi et al. (2009) and Roberts (1979) and we follow their arguments closely. At certain places new constructions are required because of the special nature of our domain. We point this out at the appropriate places.

The value difference set for any pair of distinct allocations $y, z \in D_{x}$ is defined as follows.

$$
P(y, z)=\left\{\alpha \in \mathbb{R}^{n}: \exists u \in U^{n} \text { s.t. } u(y)-u(z)=\alpha \text { and } F(u)=y\right\} .
$$

The set $P(y, z)$ is non-empty since $F$ is onto. The next claim shows that the positive quadrant starting from a point in $P(y, z)$ is always contained in $P(y, z)$.

Claim 3 If $\alpha \in P(y, z)$, and $\delta>\mathbf{0} \in \mathbb{R}^{n}$, then $\alpha+\delta \in P(y, z)$, for all distinct $y, z \in D_{x}$. 
Proof: We are given that $\alpha \in P(y, z)$. By definition of $P(y, z)$, there exists $u \in U^{n}$ such that $F(u)=y$ and $u(y)-u(z)=\alpha$. Construct $u^{\prime} \in U^{n}$ such that $u_{i}^{\prime}\left(y_{i}\right)-u_{i}\left(y_{i}\right)=\delta_{i}>u_{i}^{\prime}\left(w_{i}\right)-u_{i}\left(w_{i}\right), \forall w_{i} \neq y_{i}, z_{i}, \forall i \in N, w \in A$, where $\delta_{i}>0$ and $u_{i}^{\prime}\left(z_{i}\right)-u_{i}\left(z_{i}\right)=0, \forall i \in N$. This function $u^{\prime}$ can be constructed in exactly the same manner as $u_{i}^{\prime \prime}$ in Claim 2. By PAD-DC, $F\left(u^{\prime}\right)=y$, and by construction, $u^{\prime}(y)-u^{\prime}(z)=\alpha+\delta$. Hence $\alpha+\delta \in P(y, z)$, as needed.

Claim 4 For every $\alpha, \epsilon \in \mathbb{R}^{n}, \epsilon>\mathbf{0}$, and for all $y, z \in D_{x}$,

(a) $\alpha-\epsilon \in P(y, z) \Rightarrow-\alpha \notin P(z, y)$.

(b) $\alpha \notin P(y, z) \quad \Rightarrow \quad-\alpha \in P(z, y)$.

Proof: (a) Suppose, for contradiction, $-\alpha \in P(z, y)$. Then, there exists $u \in U^{n}$ such that $u(z)-u(y)=-\alpha$ and $F(u)=z$. Since $\alpha-\epsilon \in P(y, z)$, there exists $u^{\prime}$ such that $u^{\prime}(y)-u^{\prime}(z)=\alpha-\epsilon$ and $F\left(u^{\prime}\right)=y$. But $u^{\prime}(y)-u^{\prime}(z)<u(y)-u(z)$. This is a contradiction, since $F(u) \neq z$ according to Claim 2.

(b) ${ }^{8}$ Let $B_{y}$ and $B_{z}$ be two disjoint open balls centered at $y$ and $z$ respectively, i.e., $B_{y}=$ $\left\{k:|k-y|<\epsilon_{1}\right\}, B_{z}=\left\{k:|k-z|<\epsilon_{2}\right\}$, for some $\epsilon_{1}, \epsilon_{2}>0$. These exist since $y$ and $z$ do not match in any of their components. Choose a $u \in U^{n}$ such that $u(y)-u(z)=\alpha$ and $u_{i}\left(y_{i}\right)-u_{i}\left(w_{i}\right)>u_{i}^{y}\left(y_{i}\right)-u_{i}^{y}\left(w_{i}\right)$, for all $u^{y} \in F^{-1}(y), w \in A \backslash\left(\{y\} \cup B_{z}\right)$, and $y_{i} \neq w_{i}, \forall i \in N$. We also want $u$ to satisfy: $u_{i}\left(z_{i}\right)-u_{i}\left(w_{i}\right)>u_{i}^{z}\left(z_{i}\right)-u_{i}^{z}\left(w_{i}\right)$, for all $u^{z} \in F^{-1}(z), w \in A \backslash\left(\{z\} \cup B_{y}\right)$, and $z_{i} \neq w_{i}, i \in N$. The function $u$ can be constructed in exactly the same way as $u_{i}^{\prime \prime}$ in Claim 2 .

We now argue that $F(u) \in\{y, z\}$. If not, then $F(u)$ either belongs to $A \backslash\left(\{y\} \cup B_{z}\right)$ or $A \backslash\left(\{z\} \cup B_{y}\right)$. In both cases, Claim 2 and the earlier inequalities imply $F(u) \neq w$, which is a contradiction. Since we have shown $F(u) \in\{y, z\}$ and $u(y)-u(z)=\alpha \notin P(y, z)$, we have $F(u)=z$. Since $u(z)-u(y)=-\alpha$, it follows that $-\alpha \in P(z, y)$.

The next result shows the relationship between the sum of the interiors of two value difference sets with that of a third.

Claim 5 For every $\alpha, \beta, \epsilon^{\alpha}, \epsilon^{\beta} \in \mathbb{R}^{n}, \epsilon^{\alpha}, \epsilon^{\beta}>\mathbf{0}$, and $\forall y, z, w \in D_{x}$,

$$
\alpha-\epsilon^{\alpha} \in P(y, z) \text { and } \beta-\epsilon^{\beta} \in P(z, w) \Rightarrow \alpha+\beta-\left(\epsilon^{\alpha}+\epsilon^{\beta}\right) / 2 \in P(y, w) .
$$

Proof: The proof proceeds in a similar way as in part (b) of Claim 4, by successive elimination of possible allocations. We start by constructing three pairwise disjoint balls $B_{y}, B_{z}, B_{w}$ around $y, z, w$. This is possible since the points do not match in any of their components. Choose $u \in U^{n}$ such that,

\footnotetext{
${ }^{8}$ This proof is constructive and inspired by the 'flexibility' condition of valuations in Carbajal et al. (2013).
} 


$$
\begin{aligned}
& u(y)-u(z)=\alpha-\epsilon^{\alpha} / 2 \\
& u(z)-u(w)= \beta-\epsilon^{\beta} / 2 \\
& u_{i}\left(y_{i}\right)-u_{i}\left(l_{i}\right)> u_{i}^{y}\left(y_{i}\right)-u_{i}^{y}\left(l_{i}\right), \\
& \forall u^{y} \in F^{-1}(y), l \in A \backslash\left(\{y\} \cup B_{z} \cup B_{w}\right), y_{i} \neq l_{i}, i \in N \\
& \begin{aligned}
& u_{i}\left(z_{i}\right)-u_{i}\left(l_{i}\right)> u_{i}^{z}\left(z_{i}\right)-u_{i}^{z}\left(l_{i}\right), \\
& \forall u^{z} \in F^{-1}(z), l \in A \backslash\left(\{z\} \cup B_{w} \cup B_{y}\right), z_{i} \neq l_{i}, i \in N \\
& u_{i}\left(w_{i}\right)-u_{i}\left(l_{i}\right)> u_{i}^{w}\left(w_{i}\right)-u_{i}^{w}\left(l_{i}\right), \\
& \forall u^{w} \in F^{-1}(w), l \in A \backslash\left(\{w\} \cup B_{y} \cup B_{z}\right), w_{i} \neq l_{i}, i \in N
\end{aligned}
\end{aligned}
$$

Such a function $u$ can be constructed using arguments similar to those in Claim 4(b). Also, we can conclude that $F(u) \in\{y, z, w\}$. Since $\alpha-\epsilon^{\alpha} \in P(y, z)$, Equation (3) and an application of Claim 2 yields $F(u) \neq z$. Similarly, since $\beta-\epsilon^{\beta} \in P(z, w)$, Equation (4) and Claim 2 gives $F(u) \neq w$. Hence $F(u)=y$. Thus, $\alpha+\beta-\left(\epsilon^{\alpha}+\epsilon^{\beta}\right) / 2=u(y)-u(w) \in P(y, w)$, as needed.

The minimum translation factor is defined as follows:

$$
\gamma(y, z)=\inf \{p \in \mathbb{R} \mid p \mathbf{1} \in P(y, z)\} \text {, for all distinct } y, z \in D_{x} .
$$

Claim 6 For all distinct $y, z \in D_{x}, \gamma(y, z)$ exists in $\mathbb{R}$.

Proof: To show this, we need to show that the set $\{p \in \mathbb{R} \mid p \mathbf{1} \in P(y, z)\}$ is non-empty. Pick $u \in U^{n}$ such that $F(u)=y$, and let $p=\max _{i}\left\{u_{i}\left(y_{i}\right)-u_{i}\left(z_{i}\right)\right\}$. Increase all co-ordinates of $u(y)$ to obtain $u^{\prime}(y)$ such that $u_{i}^{\prime}\left(y_{i}\right)-u_{i}^{\prime}\left(z_{i}\right)=p, \forall i$. Note that this possible since $U$ is an ASV domain and the fact that both $y$ and $z$ are chosen from $D_{x}$. By PAD-DC, $F\left(u^{\prime}\right)=y$. Hence, the set $\{p \in \mathbb{R} \mid p \mathbf{1} \in P(y, z)\}$ is non-empty, i.e., $\gamma(y, z)<\infty$. Also $\{p \in \mathbb{R} \mid p \mathbf{1} \in P(y, z)\}$ is bounded from below, otherwise $P(z, y)$ would be empty (by Claim $4(\mathrm{~b}))$, i.e., $\gamma(y, z)>-\infty$. Hence, $\gamma(y, z)$ is a real number.

Claim 7 For all $y, z, w \in D_{x}$, the following holds:

(a) $\gamma(y, z)=-\gamma(z, y)$.

(b) $\gamma(y, w)=\gamma(y, z)+\gamma(z, w)$.

Proof: (a) Let $\gamma(y, z)=p^{*}$. Thus for any $\epsilon>0,\left(p^{*}+\epsilon-(\epsilon / 2)\right) \mathbf{1} \in P(y, z)$. By Claim 4, $\left(-p^{*}-\epsilon\right) \mathbf{1} \notin P(z, y)$. On the other hand, by definition of $\gamma(y, z),\left(p^{*}-\epsilon\right) \mathbf{1} \notin P(y, z)$. Therefore, Claim 4 implies $\left(-p^{*}+\epsilon\right) \mathbf{1} \in P(z, y)$. Hence, $-p^{*}=\inf \{p \mid p \mathbf{1} \in P(z, y)\}=\gamma(z, y)$, as needed.

(b) Fix $\epsilon>0$. We have $(\gamma(y, z)+\epsilon-(\epsilon / 2)) \mathbf{1} \in P(y, z)$, and $(\gamma(z, w)+\epsilon-(\epsilon / 2)) \mathbf{1} \in P(z, w)$. From Claim 5, we have $(\gamma(y, z)+\gamma(z, w)+2 \epsilon-(\epsilon / 2)) \mathbf{1} \in P(y, w)$. Since $\epsilon$ is arbitrary, we take its limit to zero to obtain, $\gamma(y, w) \leq \gamma(y, z)+\gamma(z, w)$. Exchanging $z$ and $w$, we get $\gamma(y, z) \leq \gamma(y, w)+\gamma(w, z)$. 
Since $\gamma(w, z)=-\gamma(z, w)$ (from part (a) above), we get, $\gamma(y, w) \geq \gamma(y, z)+\gamma(z, w)$. Hence, $\gamma(y, w)=\gamma(y, z)+\gamma(z, w)$, as needed.

Unlike the standard affine maximizer results (Roberts, 1979; Lavi et al., 2009) in finite setting, we require $\gamma(\cdot, \cdot)$ to satisfy a continuity property in ASV domain.

Claim 8 The minimum translation factor $\gamma(y, z)$ is continuous in both arguments.

Proof: The continuity of $\gamma(y, z)$ in $z$ is a consequence of the assumption that the valuation functions in the ASV domain are continuous. Since $\gamma(y, z)=-\gamma(z, y)$, it is also continuous in $y$.

Define the translated set $C(y, z)=P(y, z)-\gamma(y, z) \mathbf{1}=\{\alpha-\gamma(y, z) \mathbf{1} \mid \alpha \in P(y, z)\}$. Denote the 'interior' of $C(y, z)$ by $\stackrel{\circ}{C}(y, z)$, i.e.,

$$
\stackrel{\circ}{C}(y, z)=\{\alpha \in C(y, z) \mid \alpha-\epsilon \in C(y, z) \text {, for some } \epsilon>\mathbf{0}\} .
$$

Claim $9 \stackrel{\circ}{C}(y, z)=\stackrel{\circ}{C}(w, l)$, for all $y, z, w, l \in D_{x}, y \neq z$ and $w \neq l$.

Remark: Note that this result includes the cases, $\stackrel{\circ}{C}(y, z)=\stackrel{\circ}{C}(w, z)=\stackrel{\circ}{C}(w, y)=\stackrel{\circ}{C}(z, y)$. Therefore, Claim 9 holds even when $y, z, w, l$ are not all distinct.

Proof: Claim 5 yields the following:

$$
\begin{aligned}
\stackrel{\circ}{P}(y, z) \subseteq \stackrel{\circ}{P}(y, l)-\beta, \quad \forall \beta \in \stackrel{\circ}{P}(z, l) \\
\stackrel{\circ}{P}(z, l) \subseteq \stackrel{\circ}{P}(y, l)-\alpha, \quad \forall \alpha \in \stackrel{\circ}{P}(y, z)
\end{aligned}
$$

Substituting $y$ for $z$, and $w$ for $y$ in the second equation above, we get:

$$
\stackrel{\circ}{P}(y, l) \subseteq \stackrel{\circ}{P}(w, l)-\alpha, \quad \forall \alpha \in \stackrel{\circ}{P}(w, y)
$$

This implies,

$$
\stackrel{\circ}{P}(y, z) \subseteq \stackrel{\circ}{P}(w, l)-(\alpha+\beta) \text {, where } \alpha \in \stackrel{\circ}{P}(w, y), \beta \in \stackrel{\circ}{P}(z, l) .
$$

In particular, this is true for $\alpha=(\gamma(w, y)+\epsilon) \mathbf{1}$, and $\beta=(\gamma(z, l)+\epsilon) \mathbf{1}$, where $\epsilon>0$. Since, $\epsilon$ can be made arbitrarily small, it follows that:

$$
\begin{aligned}
\stackrel{\circ}{P}(y, z) & \subseteq \stackrel{\circ}{P}(w, l)-(\gamma(w, y)+\gamma(z, l)) \mathbf{1} \\
& =\stackrel{\circ}{P}(w, l)-(\gamma(w, y)+\gamma(y, l)-\gamma(y, l)+\gamma(z, l)) \mathbf{1} \\
& =\stackrel{\circ}{P}(w, l)-(\gamma(w, y)+\gamma(y, l)+\gamma(l, y)+\gamma(z, l)) \mathbf{1} \\
& =\stackrel{\circ}{P}(w, l)-(\gamma(w, l)+\gamma(z, y)) \mathbf{1} \\
\Rightarrow \quad \stackrel{\circ}{P}(y, z)-\gamma(y, z) \mathbf{1} & \subseteq \stackrel{\circ}{P}(w, l)-\gamma(w, l) \mathbf{1}
\end{aligned}
$$

In the second and third equalities and in the final step above, we have used Claim 7. This proves $\stackrel{\circ}{C}(y, z) \subseteq \stackrel{\circ}{C}(w, l)$. Swapping the allocations $y$ with $w$, and $z$ with $l$ and redoing the analysis, we conclude that $\stackrel{\circ}{C}(y, z) \supseteq \stackrel{\circ}{C}(w, l)$. Therefore $\stackrel{\circ}{C}(y, z)=\stackrel{\circ}{C}(w, l)$, as needed.

In view of this claim, we denote $C_{x}=\stackrel{\circ}{C}(y, z)=\stackrel{\circ}{C}(w, l)$. Our next claim shows that this set is also independent of $x$. 
Claim $10 C_{x}=C_{y}, \forall x, y \in A$.

Proof: Construct two points in $D_{x} \cap D_{y}$. Pick the first point $z_{1}$ such that all its components are different from the corresponding components of both $x$ and $y$. This is possible to pick since both $x$ and $y$ have finite number of components and both $D_{x}$ and $D_{y}$ are maximal sets. The second point $z_{2}$ is picked such that all its components are different from the corresponding components of both $x, y$ and $z_{1}$. The argument of existence follows similarly. Therefore, we conclude that $C_{x}=\stackrel{\circ}{C}\left(z_{1}, z_{2}\right)=C_{y}$, as needed.

In view of Claim 10, the subscript in $C_{x}$ can be removed. The next claim shows that this set is convex.

Claim $11 C$ is convex.

Proof: Pick distinct $\alpha, \beta \in C$. We will show $(\alpha+\beta) / 2 \in C$. Since $C$ is open, it follows that $C$ is convex. Fix distinct $y, z, w \in D$. By definition, $\alpha \in \stackrel{\circ}{P}(y, z)-\gamma(y, z) \mathbf{1}$ and $\beta \in \stackrel{\circ}{P}(z, w)-\gamma(z, w) \mathbf{1}$. Therefore, $\alpha+\beta \in(\stackrel{\circ}{P}(y, z)+\stackrel{\circ}{P}(z, w))-(\gamma(y, z) \mathbf{1}+\gamma(z, w) \mathbf{1}) \subseteq \stackrel{\circ}{P}(y, w)-\gamma(y, w) \mathbf{1}$ (using Claims 5 and 7). Hence, $\alpha+\beta \in C$.

We show that if $\alpha \in C$, then $\alpha / 2 \in C$. Suppose not, i.e., $\alpha / 2 \notin \operatorname{int}(C(w, z))$ for some $w, z \in D_{x}$ and $x \in A$. It can be either $\alpha / 2 \notin C(w, z)$ or $\alpha / 2 \in b d(C(w, z))^{9}$. Therefore, for all $\delta \geq \mathbf{0}, \delta \neq \mathbf{0}$, we have $\alpha / 2-\delta \notin C(w, z)$. An immediate consequence of Claim 4 is $-\alpha / 2+\delta \in C(z, w)$. Since $\alpha \in C$, there exists $\epsilon^{\alpha}>\mathbf{0}$ such that $\alpha-\epsilon^{\alpha} \in C(y, z)$ for some $y, z \in D_{x}$. Since $-\alpha / 2+\delta \in C(z, w)$ and $\delta$ is arbitrary, we can choose $\delta=\epsilon^{\alpha} / 4$ so that $-\alpha / 2+\epsilon^{\alpha} / 2-\epsilon^{\alpha} / 4 \in C(z, w)$. Hence, we conclude from Claim 5 that $\alpha+(-\alpha / 2)-\epsilon^{\alpha} / 8 \in C(y, w)$, i.e., $\alpha / 2 \in C$. But this is a contradiction.

Since $C=\stackrel{\circ}{C}(y, z)=\stackrel{\circ}{C}(z, y)$ is convex and $\stackrel{\circ}{C}(y, z)=(c l(-\stackrel{\circ}{C}(z, y)))^{c}$ (Claim 4$)^{10}$, it follows that $C$ is a convex half-space. Moreover, $\mathbf{0} \notin C$. Applying the separating hyperplane theorem, it follows that there exists $w \in \mathbb{R}_{\geq 0}^{n} \backslash\{\mathbf{0}\}$ such that, $w^{\top} \alpha \geq 0$, for all $\alpha \in \operatorname{cl}(C)$.

Construction of the $\kappa(\cdot)$ function Fix an arbitrary $x_{0} \in A$.

$$
\kappa(x)= \begin{cases}\gamma\left(x_{0}, x\right) \cdot w^{\top} \mathbf{1} & \text { if } x_{0} \in D_{x} \backslash\{x\} \\ \lim _{x_{n} \rightarrow x_{0}, x_{n} \in D_{x}, \forall n} \gamma\left(x_{n}, x\right) \cdot w^{\top} \mathbf{1} & \text { if } x_{0} \notin D_{x} \\ 0 & \text { if } x=x_{0}\end{cases}
$$

This function is well defined since $\gamma(y, z)$ is continuous in both arguments (Claim 8) and $D_{x}$ is dense in $A$. Hence every allocation $x_{0} \in A$ is either an element of $D_{x}$ or a limit point of $D_{x}$. Therefore, if $F(u)=x$, we have $w^{\top}(u(x)-u(y)-\gamma(x, y) \mathbf{1}) \geq 0$ for all $y \in D_{x}$. We consider two cases. If

\footnotetext{
${ }^{9} b d(S)$ denotes the boundary of $S$.

${ }^{10} \mathrm{cl}(S)$ and $S^{c}$ denote the closure and complement of $S$ respectively.
} 
$x_{0} \in D_{x}$,

$$
\begin{aligned}
& w^{\top}\left(u(x)-u(y)-\left(\gamma\left(x, x_{0}\right)+\gamma\left(x_{0}, y\right)\right) \mathbf{1}\right) \geq 0 \\
\Rightarrow & w^{\top} u(x)+\gamma\left(x_{0}, x\right) w^{\top} \mathbf{1} \geq w^{\top} u(y)+\gamma\left(x_{0}, y\right) w^{\top} \mathbf{1} \\
\Rightarrow & w^{\top} u(x)+\kappa(x) \geq w^{\top} u(y)+\kappa(y) .
\end{aligned}
$$

If $x_{0} \notin D_{x}$,

$$
\begin{array}{ll} 
& w^{\top}\left(u(x)-u(y)-\left(\gamma\left(x, x_{n}\right)+\gamma\left(x_{n}, y\right)\right) \mathbf{1}\right) \geq 0, \text { for some } x_{n} \in D_{x} \\
\Rightarrow & w^{\top} u(x)+\gamma\left(x_{n}, x\right) w^{\top} \mathbf{1} \geq w^{\top} u(y)+\gamma\left(x_{n}, y\right) w^{\top} \mathbf{1} \\
\Rightarrow & w^{\top} u(x)+\lim _{x_{n} \rightarrow x_{0}, x_{n} \in D_{x}, \forall n} \gamma\left(x_{n}, x\right) w^{\top} \mathbf{1} \geq w^{\top} u(y)+\lim _{x_{n} \rightarrow x_{0}, x_{n} \in D_{x}, \forall n} \gamma\left(x_{n}, y\right) w^{\top} \mathbf{1} \\
\Rightarrow & w^{\top} u(x)+\kappa(x) \geq w^{\top} u(y)+\kappa(y) .
\end{array}
$$

Hence we have shown that if $F(u)=x$,

$$
w^{\top} u(x)+\kappa(x) \geq w^{\top} u(y)+\kappa(y), \forall y \in D_{x} .
$$

Since $D_{x}$ is dense in $A$, each allocation $z \in A$ is either a member of $D_{x}$ or its limit point. Claim 8 implies that $\kappa$ is a continuous function. Let $\left\{y_{n}\right\} \rightarrow z$ where $y_{n} \in D_{x}, \forall n$. Thus Equation (6) holds for every $y_{n}$. Taking limits we conclude that,

$$
w^{\top} u(x)+\kappa(x) \geq w^{\top} u(z)+\kappa(z), \forall z \in A .
$$

This concludes the proof.

Q.E.D.

\section{Revenue Equivalence}

In this section, we show that the SCF $F$ also satisfies revenue equivalence. This is an observation based on the definition of revenue equivalence and a standard result (Rockafellar, 1997). The following definition of revenue equivalence is standard in literature.

Definition 8 (Revenue Equivalence) An SCF F satisfies revenue equivalence if for any two payment rules $p$ and $p^{\prime}$ that make $F$ strategyproof, there exist functions $\alpha_{i}: U^{n-1} \rightarrow \mathbb{R}$, such that,

$$
p_{i}\left(u_{i}, u_{-i}\right)=p_{i}^{\prime}\left(u_{i}, u_{-i}\right)+\alpha_{i}\left(u_{-i}\right), \forall u_{i} \in U, \forall u_{-i} \in U^{n-1}, \forall i \in N .
$$

This essentially ensures that any two payment rules that make $F$ strategyproof differ only by a factor which is independent of individual agents' valuations. The following result shows that convexity and linearity of valuations is sufficient for a strategyproof SCF to satisfy revenue equivalence. 
Theorem 2 (Rockafellar (1997); Krishna and Maenner (2001)) If the type space is convex and the valuations are linear in type, then a strategyproof SCF satisfies revenue equivalence.

In our setting, since $U$ is the ASV domain, it is clearly convex. Also the types of the agents are their valuations, which implies, trivially, that the valuations are linear in its type. Hence, the above result on revenue equivalence holds for $F$ and we conclude that $F$ satisfies revenue equivalence.

\section{A More General Result}

Our affine maximizer result rests on the fact that the allocation space is rich enough to admit a maximal and dense subset $D_{x}$ for each allocation $x$ where selfish valuations generate sufficiently rich value differences. A generalization of this result yields the following theorem, the proof of which is omitted here.

Theorem 3 (Affine Maximizers for Selfish Valuations) Let the space of allocations A be separable into components for each agent and be compact. If there exists at least one collection of $M D$ DCSs $\left\{D_{x} \mid x \in A\right\}$ such that for all $x, y \in A,\left|D_{x} \cap D_{y}\right| \geq 2$, then an onto, ANB and strategyproof $S C F F: U^{n} \rightarrow A$ is an affine maximizer.

\section{Conclusions}

From the results of this paper, it appears that in order to prove that strategyproof SCFs are affine maximizers, perhaps a certain amount of richness is required, which may be split across the set of valuations and allocations. We considered a sub-domain of the unrestricted valuations, namely the domain of selfish valuations but a "rich" set of allocations to prove our affine maximizer result.

An interesting open question is whether our results extend to more restricted domains, e.g., domains with increasing valuations.

\section{REFERENCES}

Carbajal, J. C., A. Mclennan, and R. Tourky (2013): "Truthful implementation and preference aggregation in restricted domains," Journal of Economic Theory, 148, 1074-1101.

Ching, S. (1994): "An alternative characterization of the uniform rule," Social Choice and Welfare, 11, 131-136.

Cho, W. J. And W. Thomson (2013): "On the Extension of the Uniform Rule to More Than One Commodity: Existence and Maximality Results," .

DobZinski, S. And N. NisAn (2009): "A modular approach to Roberts' theorem," in Algorithmic Game Theory, Springer, 14-23.

Goswami, M. P., M. Mitra, And A. Sen (2013): "Strategy-proofness and Pareto-efficiency in quasi-linear exchange economies," Theoretical Economics. 
Krishna, V. And E. Maenner (2001): "Convex potentials with an application to mechanism design," Econometrica, 69, 1113-1119.

Lavi, R., A. Mu'Alem, and N. Nisan (2003): "Towards a characterization of truthful combinatorial auctions," in Proceedings of 44th Annual IEEE Symposium on Foundations of Computer Science (FOCS 2003), IEEE, 574-583.

(2009): "Two simplified proofs for Roberts' theorem," Social Choice and Welfare, 32, 407423.

Marchant, T. And D. Mishra (2014): "Mechanism design with two alternatives in quasi-linear environments," Social Choice and Welfare, 1-23, forthcoming.

Maya, A. And N. Nisan (2012): "Incentive Compatible Two Player Cake Cutting," in Proceedings of the 8th International Conference on Internet and Network Economics, Springer-Verlag, WINE'12, 170-183.

Meyer-ter Vehn, M. And B. Moldovanu (2002): "Ex-post implementation with interdependent valuations," Tech. rep., Sonderforschungsbereich 504, Universität Mannheim \& Sonderforschungsbereich 504, University of Mannheim.

Mishra, D. And A. Quadir (2014): "Non-bossy single object auctions," Economic Theory Bulletin, forthcoming.

Mishra, D. AND A. SEn (2012): "Roberts' theorem with neutrality: A social welfare ordering approach," Games and Economic Behavior, 75, 283-298.

Momi, T. (2013): "Note on social choice allocation in exchange economies with many agents," Journal of Economic Theory, 148, 1237-1254.

Morimoto, S., S. Serizawa, And S. Ching (2013): "A characterization of the uniform rule with several commodities and agents," Social Choice and Welfare, 40, 871-911.

PÁPAI, S. (2000): "Strategyproof assignment by hierarchical exchange," Econometrica, 68, 14031433.

Roberts, K. (1979): The Characterization of Implementable Choice Rules, North Holland Publishing, chap. Aggregation and Revelation of Preferences, 321-348.

Rockafellar, R. T. (1997): Convex analysis, vol. 28, Princeton university press.

Sahasrabudhe, A. AND K. KAR (2008): "Bandwidth allocation games under budget and access constraints," in Information Sciences and Systems, 2008. CISS 2008. 42nd Annual Conference on, IEEE, 761-769.

Satterthwaite, M. A. And H. Sonnenschein (1981): "Strategy-proof allocation mechanisms at differentiable points," The Review of Economic Studies, 48, 587-597. 
Sprumont, Y. (1991): "The division problem with single-peaked preferences: a characterization of the uniform allocation rule," Econometrica, 59, 509-519.

Svensson, L.-G. (1999): "Strategy-proof allocation of indivisible goods," Social Choice and Welfare, $16,557-567$.

Thomson, W. (2014): "On the axiomatics of resource allocation: Interpreting nonbossiness," Mimeo.

Wang, C., Y. Yuan, C. Wang, X. Hu, and C. Zheng (2012): "Virtual bandwidth allocation game in data centers," in Information Science and Technology (ICIST), 2012 International Conference on, IEEE, 682-685.

Yaïche, H., R. R. Mazumdar, and C. Rosenberg (2000): "A game theoretic framework for bandwidth allocation and pricing in broadband networks," IEEE/ACM Transactions on Networking (TON), 8, 667-678.

\section{APPENDIX}

\section{Proof of Claim 1}

Proof: Pick an arbitrary onto affine maximizer and a tie-breaking rule $t$, i.e., $t: A \rightarrow \mathbb{R}$ is an injective mapping. The affine maximizer together with the tie-breaking rule produces an SCF $F$ in a natural way: at every valuation profile the tie-breaking rule picks a unique allocation from the set of affine maximizers. We can use standard arguments in the literature to show that this SCF is strategyproof. In particular, the following payment rule implements this SCF. For every $i \in N$ :

$$
p_{i}\left(u_{i}, u_{-i}\right)= \begin{cases}\frac{1}{w_{i}}\left(\sum_{j \neq i} w_{j} u_{j}(F(u))+\kappa(F(u))+h_{i}\left(u_{-i}\right)\right), & w_{i}>0 \\ 0 & w_{i}=0\end{cases}
$$

Where $h_{i}$ is any arbitrary function of the valuations of all the agents except agent $i$. We claim that $F$ is ANB.

Assume for contradiction that $F$ is bossy. Therefore, there exists some agent $i$ such that $x=\left(x_{i}, x_{-i}\right)=F\left(u_{i}, u_{-i}\right) \in \mathcal{A}\left(u_{i}, u_{-i}\right)$, where $\mathcal{A}\left(u_{i}, u_{-i}\right)$ is the set of affine maximizers when the valuation profile is $\left(u_{i}, u_{-i}\right)$, and $y=\left(x_{i}, y_{-i}\right)=F\left(u_{i}^{\prime}, u_{-i}\right) \in \mathcal{A}\left(u_{i}^{\prime}, u_{-i}\right)$. Now, from the definition of affine maximizer when the valuation profile is $\left(u_{i}, u_{-i}\right)$, we have:

$$
\begin{aligned}
w_{i} u_{i}\left(x_{i}\right) & +\sum_{j \neq i} w_{j} u_{j}\left(x_{j}\right)+\kappa(x) \geq w_{i} u_{i}\left(x_{i}\right)+\sum_{j \neq i} w_{j} u_{j}\left(y_{j}\right)+\kappa(y) \\
\Rightarrow & \sum_{j \neq i} w_{j} u_{j}\left(x_{j}\right)+\kappa(x) \geq \sum_{j \neq i} w_{j} u_{j}\left(y_{j}\right)+\kappa(y)
\end{aligned}
$$


Similarly, for valuation profile $\left(u_{i}^{\prime}, u_{-i}\right)$, we have:

$$
\begin{aligned}
w_{i} u_{i}^{\prime}\left(x_{i}\right) & +\sum_{j \neq i} w_{j} u_{j}\left(y_{j}\right)+\kappa(y) \geq w_{i} u_{i}^{\prime}\left(x_{i}\right)+\sum_{j \neq i} w_{j} u_{j}\left(x_{j}\right)+\kappa(x) \\
\Rightarrow & \sum_{j \neq i} w_{j} u_{j}\left(y_{j}\right)+\kappa(y) \geq \sum_{j \neq i} w_{j} u_{j}\left(x_{j}\right)+\kappa(x)
\end{aligned}
$$

Hence, we get:

$$
\sum_{j \neq i} w_{j} u_{j}\left(y_{j}\right)+\kappa(y)=\sum_{j \neq i} w_{j} u_{j}\left(x_{j}\right)+\kappa(x)
$$

Thus $x, y \in \mathcal{A}\left(u_{i}^{\prime}, u_{-i}\right)$ and $x, y \in \mathcal{A}\left(u_{i}, u_{-i}\right)$. However, this contradicts the definition of the tie-breaking rule $t$. If $x$ is chosen over $y$ at the valuation profile $\left(u_{i}, u_{-i}\right)$, then $y$ cannot be chosen over $x$ at the valuation profile $\left(u_{i}^{\prime}, u_{-i}\right)$.

\section{Proof of Proposition 1}

Define a partial order $\succsim$ on $\mathscr{D}_{x}$ as follows. For all $E_{x}, E_{x}^{\prime} \in \mathscr{D}_{x}, E_{x} \succsim E_{x}^{\prime} \Leftrightarrow E_{x}^{\prime} \subseteq E_{x}$.

LEMma $2\left(\mathscr{D}_{x}, \succsim\right)$ has a maximal element.

Proof: Consider an arbitrary chain, i.e., a linearly ordered subclass, in $\left(\mathscr{D}_{x}, \succsim\right)$, say $(\mathcal{B}, \succsim \mid \mathcal{B})$. By definition, $\mathcal{B} \subseteq \mathscr{D}_{x}$ and $\left.\succsim\right|_{\mathcal{B}}$ is a restriction of $\succsim$ to $\mathcal{B}$, which is complete and anti-symmetric. Consider $E^{\mathcal{B}}=\cup_{E \in \mathcal{B}} E$.

We claim that $E^{\mathcal{B}} \in \mathscr{D}_{x}$. Suppose not. Then $\exists x, y \in E^{\mathcal{B}}, x \neq y$ such that $x$ and $y$ are same at least in one component. By definition of $E^{\mathcal{B}}$ and considering the fact that $\left(\mathcal{B},\left.\succsim\right|_{\mathcal{B}}\right)$ is a chain, it must be true that $\exists E^{x, y} \in \mathcal{B}$ such that $x, y \in E^{x, y}$. This contradicts the fact $\mathcal{B} \subseteq \mathscr{D}_{x}$.

Notice that $E^{\mathcal{B}} \succsim E, \forall E \in \mathcal{B}$. Hence every chain in $\left(\mathscr{D}_{x}, \succsim\right)$ has an upper bound in $\mathscr{D}_{x}$. By Zorn's lemma, $\left(\mathscr{D}_{x}, \succsim\right)$ has a maximal element.

Lemma 3 There exists a $D \in \mathscr{D}_{x}$ such that $D$ is dense in $A$.

Proof sketch: The proof is constructive. We first prove a claim that is used in the construction. Define a grid as a collection of axis-parallel planes. Hence a grid splits the space $A$ into several cells. A open grid cell profile is the collection of the open cells induced by the grid (see Figure 3 for an illustration in two dimensions).

Claim 12 For each finite open grid cell profile $G$ of $A$, there exists $C_{G} \subseteq A$ such that,

(i) each cell of $G$ has exactly one point of $C_{G}$,

(ii) $C_{G}$ is a DCS. 


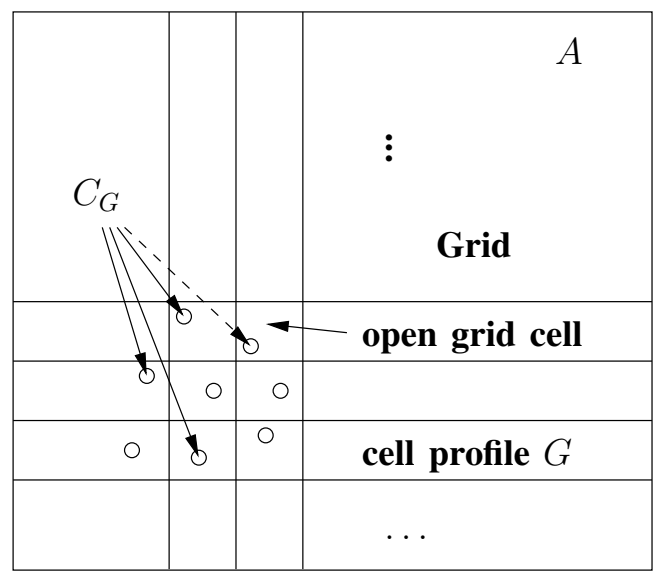

Figure 3: Illustration for the proof of Lemma 3.

Proof sketch: Define a random experiment which selects one point uniformly at random for every open cell in the cell profile $G$, independent from every other cell. It is clear that it is a measure zero event to pick two points which have at least one identical coordinate. Hence, there exists at least one configuration which is a DCS.

We start with $x$ and call $D_{1}=\{x\}$. Draw axis parallel planes from $x$ so that it divides $A$ into a finite open grid cell profile $G_{1}$. Pick a DCS $C_{G_{1}}$. This is possible by the above claim. Note that $\{x\} \cup C_{G_{1}}$ is also a DCS. Let us call these set of points $D_{2}$. Now we draw axis parallel planes from each of the points in $D_{2}$, consider the corresponding finite grid cell profile $G_{2}$ and pick a DCS $C_{G_{2}}$ and construct $D_{3}$ in a similar way. We claim that $D:=\cup_{i=1}^{\infty} D_{i}$ is dense in $A$. Clearly $D \in \mathscr{D}_{x}$ by construction. It is easy to show that for every $z \in A$ and $\epsilon>0$, there exists a $n_{\epsilon}$, large enough, such that $\exists y \in D_{n_{\epsilon}}$ having $\|y-z\|<\epsilon$. This concludes the proof.

Pick a $D \in \mathscr{D}_{x}$ dense in $A$. Define, $\chi_{D}=\left\{E_{x} \in \mathscr{D}_{x} \mid E_{x} \succsim D\right\}$. It is clear that every element of $\chi_{D}$ is dense in $A$. Also $D \in \chi_{D}$, therefore, $\chi_{D} \neq \emptyset$.

LEMma $4\left(\chi_{D},\left.\succsim\right|_{\chi_{D}}\right)$ has a maximal element.

The argument of the proof of this lemma is similar to Lemma 2. We show that this maximal element is a maximal element in the set of all DCSs.

LEMma 5 Every maximal element of $\left(\chi_{D}, \succsim \mid \chi_{\chi_{D}}\right)$ is a maximal element of $\left(\mathscr{D}_{x}, \succsim\right)$.

Proof: Suppose not. Fix a maximal element $\theta_{\chi_{D}}$ of $\left(\chi_{D},\left.\succsim\right|_{\chi_{D}}\right)$ that is not maximal in $\left(\mathscr{D}_{x}, \succsim\right)$. Therefore, there exists some $\theta \in \mathscr{D}_{x}$ such that $\theta \succ \theta_{\chi_{D}}$. By definition, $\theta_{\chi_{D}} \succsim D$. By transitivity of order relation $\theta \succ D$, which implies $\theta \in \chi_{D}$. This contradicts the maximality of $\theta_{\chi_{D}}$ in $\left(\chi_{D},\left.\succsim\right|_{\chi_{D}}\right)$.

Hence, there exists a maximal element of $\mathscr{D}_{x}$ which is dense in $A$, as needed. 